\title{
C-graphs - A mixed graphical representation of groups
}

\author{
SINU N.VIJAYAN \\ Department of Mathematics \\ ANJALY KISHORE \\ Department of Mathematics \\ St.Mary's College, Thrissur, Kerala \\ Vimala College, Thrissur, Kerala \\ INDIA \\ INDIA
}

Abstract: Corresponding to each group $\Gamma$, a mixed graph $G=\left(\Gamma, E, E^{\prime}\right)$ called C-graph is assigned, such that the vertex set of $G$ is the group itself. Two types of adjacency relations, that is, one way and two way communication is defined for vertices, to get a clear idea of the underlying group structure. An effort to answer the question, 'Is there any relation between the order of an element in the group and degrees of the corresponding vertex in the C-graph', by proposing a mathematical formula connecting them is made. Established an upper bound for the total number of edges in a C-graph $G$. For a vertex $z$ in $G$, the concept Connector Edge $C E_{z}$ is defined, which convey some structural properties of the group $\Gamma$. The Connector Edge Set is defined for both a vertex $z$ and the whole C-graph $G$, and is denoted as $\mathscr{C} \mathscr{E}_{z}$ and $\mathscr{C} \mathscr{E}_{G}$ respectively. Proposed the result, $\mathscr{C} \mathscr{E}_{G}=E$ if and only if $|\Gamma|=2^{n}, n \in \mathbb{N}$. Finally, the properties of $G$, which the Connector Edge Set $\mathscr{C} \mathscr{E} G$ carry out are discussed.

Key-Words: C-graph, Group, Mixed graph, Connector Edge, Connector Edge Set

Received: March 30, 2021. Revised: October 5, 2021. Accepted: October 20, 2021. Published: November 3, 2021.

\section{Introduction}

Combining different branches of Mathematics and advancing applications of one to the other is a vast area for research [Eg : Topological graph theory, Algebraic topology, Algebraic graph theory and so on]. The main objective of this paper is, the study of such an interconnection. As the title suggests, the study is between Graph theory and Group theory, and method of study is, represent every group by a corresponding graph structure called C-graph, and then discuss the algebraic properties of the group by corresponding graph representation.

The key point which is going to differentiate the C-graph from existing graphs on groups is, it is a Mixed graph [infact no mixed graph is defined for an algebraic structure] and it gives more transparency for the relation between the elements of the group, as in the way a mixed graph interpret the communication between nodes or vertices by characterizing the edges as directed and undirected.

At the same time, both directed as well as undirected edges are dealt with simultaneously here. Thus, before entering into the definition and properties of C-graph, it is better to go through some of the existing graphs on Algebraic structures. The next section summerizes such studies.

\section{Graphs on Algebraic structures}

Cayley graph[1] is a significant graphical representation of groups introduced by A.Cayley in 1878 for the purpose of constructing a pictorial representation of finite groups. For each generating set $S$ of a finite group $G$, there is a directed graph representing the group in terms of the generators in $S$ and called it as Cayley graph of $G$ with respect to $S$. Bretto[2] in 2007 pointed out some of the limitations of Cayley graph such as it is regular always and do not give much informa- 
tion about the group and so on, and then defined G-graph as a solution for this according to him. Also, he presented an algorithm for the construction of G-graphs from groups.

Neumann [3] in 1976 defined Commutinggraph of a group, as a graph with vertex set the group itself and two vertices $g, h$ are joined by an undirected edge if and only if they do not commute as elements of $G$. Beck[4], in 1988 introduced the idea of a Zero divisor graph of a commutative ring $R$ with identity, in which he make two vertices $x, y$ adjacent if and only if $x y=0$.

Abdollahi[5] et.al. assigned the Non commuting graph to an arbitrary non abelian group, the graph is obtained by taking elements not in center of the group and make two elements adjacent if they do not commute. Kelarev and Quinn [6] defined Directed power graph $\operatorname{Pow}(S)$ of a semigroup $S$ as a graph with vertex set $S$ itself and for two distinct elements $u, v$ of $S$ if $v$ is a power of $u$, then $(u, v)$ is a directed edge. Divisibility graph and Annhilator graph of a semigroup $S$ are also defined by Kelarev and Quinn[7]. The Divisibility graph $\operatorname{Div}(S)$ has vertex set $S$ and edges $(u, v)$, where $u \neq v$ and $u$ divides $v$. The Annhilator graph $\operatorname{Ann}(S)$ of $S$ with 0 , has vertex set $S$ and edge set $\{(u, v) \in S \times S: u v=0, u \neq v\}$.

Chakrabarty[8] et.al. investigated the Undirected power graph of a semigroup $S$, where the graph have vertex set $S$ and two distinct elements $a$ and $b$ of $S$ are adjacent if and only if one is a positive power of the other. Also, they observed that power graph is connected for any finite group and is complete if and only if the group is cyclic of order 1 or $p^{m}$, where $p$ is a prime.

Redmond[9] in 2002 defined zero divisor graph corresponding to a non commutative ring in four different ways. Among them one is a directed graph and others are undirected in nature. The total graph of a commutative ring $R$ was introduced by Anderson and Badawi[10][11] in 2008, as a graph with vertex set $R$ and two distinct vertices $x$ and $y$ of $R$, are adjacent only if $x+y \in$ $Z(R)$.

Smarandache and Vasantha[12] in 2009, defined the identity graph for a semigroup $S$, in which the vertex set is the semigroup itself and two distinct vertices $x$ and $y$ are adjacent if and only if $x y$ is the identity in $S$. Then these graphs are extednded to commutative rings, groups and multigroups also by different authors[12][13][14]. Erfanian and Tolue[15] in 2012 associated a Relative non nil-n graph with a group $G$ which is not a nil-n group. The vertex set of this graph is $G \backslash z_{n}(G)$ and two distinct vertices $x$ and $y$ are adjacent if $[x, y] \notin z_{n-1}(G)$, where $z_{n}(G)$ is the $n^{\text {th }}$ term of the upper central series of $G$.

Niroomand[16] in 2017 introduced the Non exterior square graph corresponding to non cyclic groups, in which two distinct vertices $x, y \in$ $G \backslash \hat{Z}(G)$ join by an edge if $x \wedge y \neq 1$. Akbari et.al[17][18][19] in 2017 defined enhanced power graph of groups as a graph with vertex set $G$ itself and two distinct vertices $x$ and $y$ of $G$ are adjacent if they are in the same cyclic subgroups. In 2019, Banerjee[20] [21] assigned a new graph on finite group and named it as Coprime graph, with vertex set as the group itself and two distinct vertices $x$ and $y$ of the graph are adjacent only if the gcd of orders of $x$ and $y$ is either 1 or a prime.

So far discussed papers and their continued works are focusing on the investigation of algebraic structure of the group using associated group. Now, more innovative results are being proposed by different researchers on this field all around the world. In our paper, a mixed graphical representation of groups called C-graph is introduced and studied some of its properties.

\section{Basic definitions}

Mixed graph is a graph containing oriented as well as unoriented set of edges. That is, a mixed graph $G=\left(V, E, E^{\prime}\right)$ is a graph contain- 
ing un-oriented $E$, as well as oriented $E^{\prime}$, set of edges. Two mixed graphs $G_{1}$ and $G_{2}$ are isomorphic[22], if there is a one-one correspondence between their vertex set which preserves adjacency. That is, there exist a one-one and onto function $f: V\left(G_{1}\right) \longrightarrow V\left(G_{2}\right)$ such that for any two vertices $u$ and $v$ of $G_{1}$, there exist two vertices $f(u)$ and $f(v)$ in $G_{2}$, with the property that, if there is a directed edge from $u$ to $v$ or a directed edge from $v$ to $u$, then there is a directed edge from $f(u)$ to $f(v)$ or a directed edge from $f(v)$ to $f(u)$ respectively. Also, if $u$ and $v$ are connected by an undirected edge then so are $f(u)$ and $f(v)$. A group in which every non identity element is of order 2 is called a Boolean group or Abelian 2group. That is, every element in a Boolean group is self inverse and every Boolean group is abelian.

Throughout this paper, $\Gamma$ is a group and $G$ is a graph. $Z_{n}$ is the set of all possible remainders when an integer is divided by $n$. Here, we use $Z_{n}$ for the group $\left(Z_{n},+_{n}\right)$. For an element $a$ in $\Gamma, o(a)$ is the order of $a$. The identity element of the group is denoted by $i$.

\section{Structure and Properties of C-graph of a group}

In this section, a new graphical representation called C-graph of a group is introduced. The ' $\mathrm{C}$ 'in this name is for 'cyclic subgroup', since, the adjacency of vertices in the graph depends on the cyclic subgroups of the corresponding elements of the group. C-graph has a close connection with the power graph, or rather, a modification of the power graph, as in a way the communication between the vertices of the graph is more clarified. For a group $\Gamma$, in the directed power graph, for $x, y \in \Gamma$, if $\langle x\rangle=\langle y\rangle$, there are two different directed edges $x \longrightarrow y$ and $y \longrightarrow x$. Also, in the undirected power graph, there is an undirected edge between $x$ and $y$ for all the three cases, $\langle x\rangle \subset\langle y\rangle,\langle x\rangle \supset\langle y\rangle$ and $\langle x\rangle=\langle y\rangle[24]$. Thus identification of the relation between elements of the group by merely looking these corresponding graphs is not explicit.

In order to analyse the pattern of communication between nodes, that is to find which two nodes have two way communication and which two nodes have only one way communication, we attempt to define the C-graph for a group. The C-graph so defined is a mixed graph, that is, a graph with both directed and undirected edges.

\subsection{Definitions}

Definition 4.1. For a group $\Gamma$, the $\mathbf{C}$-graph $G=\left(\Gamma, E, E^{\prime}\right)$ is the graph with vertex set $V(G)=\Gamma$ and for $x, y \in \Gamma, x \neq y$,

If $\langle x\rangle \subset\langle y\rangle$, then there is a directed edge from $x$ to $y$

If $\langle x\rangle=\langle y\rangle$, then there is an undirected edge between $x$ and $y$.

where $E$ is the set of undirected edges and $E^{\prime}$ is the set of directed edges of $G$.

Note 4.1. In the C-graph $G$ of the group $\Gamma$, for any two vertices $x$ and $y$, if there is a directed edge from $x$ to $y$, then it is denoted as $x \longrightarrow y$. Also, if there is an undirected edge between $x$ and $y$, then denote it as $x y$.

\subsection{Observations}

Consider $x, y \in \Gamma$, if either $\langle x\rangle \subseteq\langle y\rangle$ or $\langle y\rangle \subseteq\langle x\rangle$ in $\Gamma$, then we can say that $\langle x\rangle$ and $\langle y\rangle$ are comparable and so are the elements $x$ and $y$.

In this context, for any two comparable elements $x$ and $y$ of a group $\Gamma$,

if $\langle x\rangle \subset\langle y\rangle$, then $\langle x\rangle$ is weaker and $\langle y\rangle$ is stronger cyclic subgroups.

If $\langle x\rangle=\langle y\rangle$, then the two cyclic subgroups are equal in strengh.

Thus, for any two comparable elements $x$ and $y$ of $\Gamma$, the element which generates weaker cyclic subgroup is the weaker element and the other is stronger. So, the power of generating more elements of the group is considered as a measure of 
strength for comparable elements.

Now, let us check how the comparability of elements of the group $\Gamma$ reflects in the corresponding C-graph $G$. Any two elements $x$ and $y$ are comparable in the group $\Gamma$ if and only if there is an edge between $x$ and $y$ in the C-graph $G$. Explicitly, there are two cases here.

For $x, y \in \Gamma$,

$x \longrightarrow y$ if and only if $x$ is weaker and $y$ is stronger in $\Gamma$.

$x y$ in $G$ if and only if $x$ and $y$ are equal in strength in $\Gamma$.

We summerize the above discussion as a chart in the Appendix.

Since $G$ is a mixed graph, for a vertex $x$ there are three types of degrees namely Degree, Indegree, and Outdegree. The first one is contributed by undirected edges and the other two by directed edges.

Degree : It is the number of undirected edges incident with $x$ and is denoted by $\operatorname{deg}(x)$.

Indegree : It is the number of directed edges with $x$ as their terminal vertex and is denoted by $\operatorname{deg}^{-}(x)$.

Outdegree : It is the number of directed edges with $x$ as their initial vertex and is denoted by $\operatorname{deg}^{+}(x)$.

Example 4.1. Given below are the C-graphs of the groups $\Gamma_{1}=S_{3}$ and $\Gamma_{2}=\mathbb{Z}_{8}$

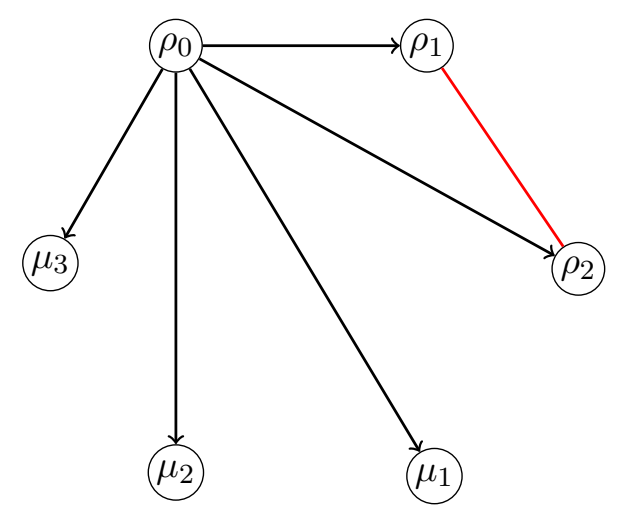

Fig.1 C-graph $G\left(S_{3}\right)$

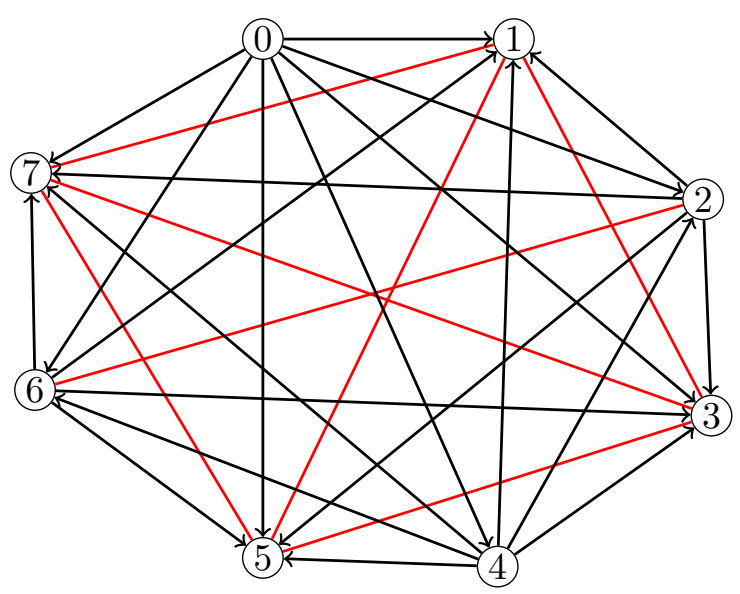

Fig.2 C-graph $G_{2}\left(\mathbb{Z}_{8}\right)$

\subsection{Properties of Degrees and Order of an element}

The main objective of this section is to discuss the problem, 'Is there any relation between the order of an element in the group and degrees of the corresponding vertex in the C-graph?'. A method is constructed to obtain the order of each element in the group from the corresponding C-graph.

Remark 4.1. For the C-graph $G=\left(\Gamma, E, E^{\prime}\right)$ of a finite group $\Gamma$ with identity $i$.

1. $\operatorname{deg}(i)=\operatorname{deg}^{-}(i)=0$.

2. $\operatorname{deg}^{+}(x)=|\Gamma|-1$ if and only if $x=i$.

Proposition 4.1. For any prime $p$, the C-graph $G$ of the finite group $\mathbb{Z}_{p}$ has the following properties.

1. $G$ contains a complete subgraph with $p-1$ vertices all of its edges are undirected.

2. $G$ consists of only $p-1$ directed edges, all of it are of the form $(0, t)$ where $t=$ $1,2,3, \ldots, p-1$.

3. $\operatorname{deg}(0)=\operatorname{deg}^{-}(0)=0, \operatorname{deg}^{+}(0)=p-1$.

4. $\operatorname{deg}(x)=p-1$, if $x \neq 0$.

5. $\operatorname{deg}^{-}(x)=1, \operatorname{deg}^{+}(x)=0$, if $x \neq 0$. 
Proof. 1. Since $p$ is a prime number, the integers $t$, such that $1 \leq t \leq p-1$, generates $\mathbb{Z}_{p}$. That is, $\langle 1\rangle=\langle 2\rangle=\langle 3\rangle=\ldots=$ $\langle p-1\rangle=\mathbb{Z}_{p}$. Which means in the C-graph $G$ of $\mathbb{Z}_{p}$, there is an undirected edge between any pair of vertices other than 0 . So, the vertices $1,2, \ldots, p-1$ induces a complete subgraph of $G$.

2. $\langle 0\rangle=\{0\}$ is a subgroup of all the other cyclic subgroups. So, in the C-graph $G$ there are $p-1$ directed edges from the vertex 0 . These are the only directed edges of $G$, since all the other cyclic subgroups are equal.

3. Clear from (1) and (2) of the remark 4.1.

4. (4) and (5) are obvious from the (1) and (2) of the same proposition.

Theorem 4.1. Let $\Gamma$ be a finite group and $G=\left(\Gamma, E, E^{\prime}\right)$ the C-graph of $\Gamma$. Then, for any $a$ in $\Gamma$, the order of $a, o(a)$ can be directly deduced from the C-graph $G$. In particular, $o(a)=\operatorname{deg}(a)+\operatorname{deg}^{-}(a)+1$.

Proof. Since C-graph is constructed by using the subset relation of cyclic subgroups of each element, it is possible to deduce the order of elements from the graph itself. For an element $a$ of $\Gamma$, define

$$
\begin{aligned}
X= & \{x \in \Gamma: x \longrightarrow a \text { is a directed } \\
& \text { edge in } G, x \neq a\} \\
Y= & \{y \in \Gamma: y a \text { is an undirected } \\
& \text { edge in } G, y \neq a\} \\
B= & \langle a\rangle-\{a\} . \\
\text { Let } b \in B \Rightarrow & b=a^{m}, b \neq a, m \in N . \\
\Rightarrow & \langle b\rangle=\left\{b^{n}: n \in N\right\}=\left\{\left(a^{m}\right)^{n}: n \in \mathbb{N}\right\} \\
& \subseteq\langle a\rangle . \\
\Rightarrow & \langle b\rangle \subseteq\langle a\rangle . \\
\Rightarrow & \text { either }\langle b\rangle \subset\langle a\rangle \text { or }\langle b\rangle=\langle a\rangle .
\end{aligned}
$$

This means either $b \longrightarrow a$ is a directed edge or $b a$ is an undirected edge in $G$. Thus, $b \in X$ or $b \in Y$. That is, $b \in X \cup Y$ and hence

$$
B \subset X \cup Y .
$$

Suppose

$$
\begin{aligned}
c \in X \cup Y & \Rightarrow c \in X \text { or } c \in Y . \\
& \Rightarrow \quad c \longrightarrow a \text { is a directed edge } \\
& \quad \text { or } c a \text { is an undirected edge. } \\
& \Rightarrow\langle c\rangle \subset\langle a\rangle \text { or }\langle c\rangle=\langle a\rangle . \\
& \Rightarrow \quad\langle c\rangle \subseteq\langle a\rangle \text { and } c \neq a . \\
& \Rightarrow \quad c \in(\langle a\rangle-\{a\}) \Longrightarrow c \in B .
\end{aligned}
$$

and hence

$$
X \cup Y \subset B .
$$

Thus from (1) and (2)

$$
B=X \cup Y .
$$

Suppose that

$$
\begin{aligned}
d \in X \cap Y \Rightarrow & d \in X \text { and } d \in Y . \\
\Rightarrow & \text { Both directed and undirected } \\
& \text { edges exist between } d \text { and } a .
\end{aligned}
$$

which is not possible. Therefore,

$$
X \cap Y=\emptyset .
$$

That is, $B$ is the disjoint union of $X$ and $Y$.

Therefore,

$$
\begin{aligned}
|B|=|X|+|Y| & \\
& \Rightarrow|\langle a\rangle-1|=\operatorname{deg}^{-}(a)+\operatorname{deg}(a) . \\
& \Rightarrow|\langle a\rangle|=\operatorname{deg}(a)+\operatorname{deg}^{-}(a)+1 \\
& \Rightarrow o(a)=\operatorname{deg}(a)+\operatorname{deg}^{-}(a)+1
\end{aligned}
$$

Illustration 4.1. Let $\Gamma_{1}=S_{3}$, then from the Cgraph $G_{1}$ of $\Gamma_{1}$ in Fig 1

$$
\begin{aligned}
& o\left(\rho_{0}\right)=0+0+1=1 \\
& o\left(\rho_{1}\right)=1+1+1=3 \\
& o\left(\rho_{2}\right)=1+1+1=3 \\
& o\left(\mu_{1}\right)=0+1+1=2 \\
& o\left(\mu_{2}\right)=0+1+1=2 \\
& o\left(\mu_{3}\right)=0+1+1=2
\end{aligned}
$$

Illustration 4.2. Let $\Gamma_{2}=\mathbb{Z}_{8}$, then from the 
C-graph $G_{2}$ of $\Gamma_{2}$ in Fig 2

$$
\begin{aligned}
& o(0)=0+0+1=1 \\
& o(1)=3+4+1=8 \\
& o(2)=1+2+1=4 \\
& o(3)=3+4+1=8 \\
& o(4)=0+1+1=2 \\
& o(5)=3+4+1=8 \\
& o(6)=1+2+1=4 \\
& o(7)=3+4+1=8
\end{aligned}
$$

Corollary 4.1. Let $\Gamma$ be a finite group and $G$ be its C-graph. For an $a \in \Gamma, o(a)=p$, a prime if and only if $\operatorname{deg}^{-}(a)=1$ in $G$. That is, only one directed edge pointed towards $a$ and which is from the identity. In that case, the order of such an element $a$ is $\operatorname{deg}(a)+2$.

Proof. For an element $a$ in $\Gamma$ to be of prime order it is necessary and sufficient that, for any $b \in \Gamma$ with $\langle b\rangle \subset\langle a\rangle \Longrightarrow b=i$. Thus, the only directed edge pointing towards $a$ is from $i$, which implies $\operatorname{deg}^{-}(a)=1$. Now, from Theorem 4.1, $o(a)=\operatorname{deg}(a)+2$.

Corollary 4.2. Two groups having isomorphic C-graphs have the same numbers of elements of equal order. Thus, isomorphism between Cgraphs preserves order property.

Proof. From Theorem 4.1, it is clear that order of each element $a$ in the group can be deduced from the corresponding $\mathrm{C}$-graph by the formula $o(a)=\operatorname{deg}(a)+\operatorname{deg}^{-}(a)+1$. So, the proof is clear.

For a mixed graph, total number of edges can be determined using the below formula.

Lemma 4.1. [22] Let $V_{m}=\left\{v_{1}, v_{2}, \ldots, v_{m}\right\}$ be the vertex set of mixed graph $G_{m}=(V, E, \bar{E})$. The total number of edges $E_{m}$ in the mixed graph $G_{m}$ is given by

$$
\left|E_{m}\right|=\frac{1}{2} \sum_{v \in V_{m}} \operatorname{deg}(v)+\sum_{v \in V_{m}} d e g^{-}(v) . \quad \text { or }
$$

$$
\left|E_{m}\right|=\frac{1}{2} \sum_{v \in V_{m}} \operatorname{deg}(v)+\sum_{v \in V_{m}} \operatorname{deg}^{+}(v) .
$$

From Lemma 4.1, the total number of directed edges and undirected edges of the C-graph can be deduced as follows.

Theorem 4.2. Let $\Gamma$ be a finite group and $G=$ $\left(\Gamma, E, E^{\prime}\right)$ be the C-graph of $\Gamma$. Then

1. $|E|=\frac{1}{2} \sum_{x \in \Gamma} \operatorname{deg}(x)$.

2. $\left|E^{\prime}\right|=\sum_{x \in \Gamma} \operatorname{deg}^{-}(x)=\sum_{x \in G} \operatorname{deg}^{+}(x)$.

Theorem 4.3. Let $\Gamma$ be a group with $n$ elements and $G$ the C-graph of $\Gamma$. Then, the total number of edges in $G$ is $|E|+\left|E^{\prime}\right| \leq \sum_{x \in \Gamma} o(x)-n$, and the equality holds for Boolean groups only.

Proof. We have from Theorem 4.1,

$$
o(x)=\operatorname{deg}(x)+\operatorname{deg}^{-}(x)+1 \quad \forall x \in \Gamma .
$$

Now, sum up over all the elements of $\Gamma$, we get $\sum_{x \in \Gamma} o(x)=\sum_{x \in \Gamma} \operatorname{deg}(x)+\sum_{x \in \Gamma} \operatorname{deg}^{-}(x)+n$. That is, $\sum_{x \in \Gamma} \operatorname{deg}(x)+\sum_{x \in \Gamma} \operatorname{deg}^{-}(x)=\sum_{x \in \Gamma} o(x)-n$ From Theorem 4.2,

Total number of edges in $G$

$$
\begin{aligned}
& =|E|+\left|E^{\prime}\right| . \\
& =\frac{1}{2} \sum_{x \in \Gamma} \operatorname{deg}(x)+\sum_{x \in \Gamma} \operatorname{deg}^{-}(x) . \\
& \leq \sum_{x \in \Gamma} \operatorname{deg}(x)+\sum_{x \in \Gamma} \operatorname{deg}^{-}(x) . \\
& =\sum_{x \in \Gamma} o(x)-n .
\end{aligned}
$$

Thus, $|E|+\left|E^{\prime}\right| \leq \sum_{x \in \Gamma} o(x)-n$.

Suppose that for a group $\Gamma$,

$$
|E|+\left|E^{\prime}\right|=\sum_{x \in \Gamma} o(x)-n
$$

That is,

$$
\begin{aligned}
\frac{1}{2} \sum_{x \in \Gamma} \operatorname{deg}(x)+\sum_{x \in \Gamma} \operatorname{deg}^{-}(x) \\
=\sum_{x \in \Gamma} \operatorname{deg}(x)+\sum_{x \in \Gamma} \operatorname{deg}^{-}(x) \\
\frac{1}{2} \sum_{x \in \Gamma} \operatorname{deg}(x)=\sum_{x \in \Gamma} \operatorname{deg}(x) \\
\frac{1}{2} \sum_{x \in \Gamma} \operatorname{deg}(x)=0 \\
\sum_{x \in \Gamma} \operatorname{deg}(x)=0 \\
\operatorname{deg}(x)=0 \quad \forall x \in \Gamma
\end{aligned}
$$

That is, there is no undirected edge in the Cgraph $G$ of the given group $\Gamma$. This means, no two distinct elements of the group $\Gamma$, generates same cyclic subgroup.

Claim : $x=x^{-1} \forall x \in \Gamma$. 
Suppose, $\exists$ an $x \in \Gamma$ such that $x^{-1}=y \neq x$, then $\langle x\rangle=\left\langle x^{-1}\right\rangle=\langle y\rangle$. that is, $\langle x\rangle=\langle y\rangle, x \neq y$, which is a contradiction. Therefore, $x=x^{-1}$ $\forall x \in \Gamma$.

$$
\begin{aligned}
& \Longrightarrow x^{2}=i \forall x \in \Gamma \\
& \Longrightarrow\langle x\rangle=\{i, x\} \forall x \in \Gamma .
\end{aligned}
$$

Thus, $\Gamma$ is a Boolean group.

Example 4.2. For $\Gamma=V$, the klien-4-group [example of a Boolean group], the C-graph $G$ of $V$ is a directed tree as below.

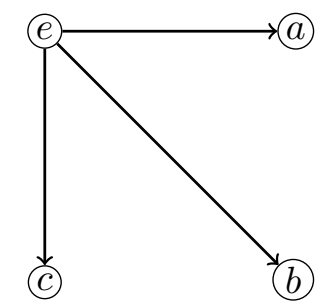

Fig.3 C-graph $G(V)$

Total number of edges of $G(V)$

$$
\begin{aligned}
& =\sum_{x \in V} o(x)-4 \\
& =o(e)+o(a)+o(b)+o(c)-4 \\
& =1+2+2+2-4 \\
& =3
\end{aligned}
$$

\section{Connector Edge}

In the C-graph $G$ of a group $\Gamma$, the concept of Connector Edge for a vertex is introduced to study the group structure using the graph theory.

Definition 5.1. Let $z$ be a vertex in the C-graph $G=\left(\Gamma, E, E^{\prime}\right)$ of a group $\Gamma$. Then $z$ is said to have a Connector Edge in $G$, if there exist two distinct vertices $x$ and $y$ both different from $z$ and an undirected edge $w$ connecting $x$ and $y$ such that $z \longrightarrow x$ and $z \longrightarrow y$ are two directed edges in $G$. Here $w$ is said to be a Connector Edge of the vertex $z$, and is denoted $w=C E_{z}$. Also, $z$ is said to be a Common Neighbour Pointing Towards (CoNPT) the vertices $x$ and $y$, and is denoted by $z=C o N P T_{x y}$.

Example 5.1. Consider $\Gamma=Q_{8}=$ $\{1,-1, i,-i, j,-j, k,-k\}$, the quartenion group, then $G\left(Q_{8}\right)$ is given below.

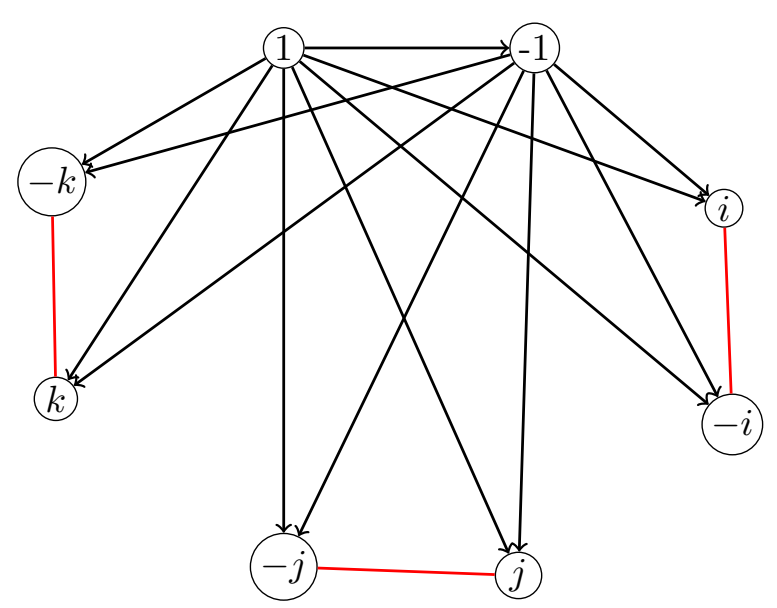

Fig.4 C-graph $G\left(Q_{8}\right)$

Here, the edge $-i i$ is a Connector Edge of the vertex -1 . That is, $-i i=C E_{-1}$ and $-1=$ $\operatorname{CoNPT}_{-i i}$

Similarly, $-i i=C E_{1}$ and $1=C o N P T_{-i i}$

$$
\begin{aligned}
& -j j=C E_{1} \text { and } 1=C o N P T_{-j j} \\
& -j j=C E_{-1} \text { and }-1=C o N P T_{-j j} \\
& -k k=C E_{1} \text { and } 1=C o N P T_{-k k} \\
& -k k=C E_{-1} \text { and }-1=C o N P T_{-k k}
\end{aligned}
$$

Note 5.1. 1. A vertex $z$ in the C-graph $G$, may have more than one Connector Edges. For example, for $G\left(Q_{8}\right)$ in example $5.1-i i$, $-j j,-k k$ are Connector Edges of the vertex -1 .

2. The notation $w=C E_{z}$ means, the edge $w$ is one of the Connector Edges of the vertex $z$. That is, this is not a unique notation.

3. For any two distinct vertices $x$ and $y$ in $G$, there may have more than one Common Neighbour Pointing Towards $x$ and $y$. For example, -1 and 1 are Common Neighbour Pointing Towards $-i$ and $i$.

4. The notation $z=\operatorname{CoNPT}_{x y}$ means the vertex $z$ is one of the Common Neighbour Pointing Towards $x$ and $y$. That is, this is not a unique notation. 
Now, let us think about the basic properties of the group that are carried out by the Connector Edges of the corresponding C-graph.

A Connector Edge for a vertex $z$ in the C-graph $G$ of a group $\Gamma$, ensures the presence of two distinct vertices $x$ and $y$ distinct from $z$ and two directed edges $z \longrightarrow x$ and $z \longrightarrow y$ and an undirected edge $x y$ in $G$. Thus, a vertex $z$ of the C-graph has a Connector Edge implies there are two distinct elements $x$ and $y$ in the corresponding group which generates the same cyclic subgroup $H=\langle x\rangle=\langle y\rangle$, properly containing the element $z$.

Definition 5.2. For a vertex $z$ in the C-graph $G$, define the set $\mathscr{C} \mathscr{E}_{z}=\left\{w \in E: w=C E_{z}\right\}$, and is called the Connector Edge Set or $C E$ Set of the vertex $z$. That is, $\mathscr{C} \mathscr{E}_{z}$ is the collection of all Connector Edges of the vertex $z$ in the C-graph $G$ of the group $\Gamma$.

Example 5.2. For $G\left(Q_{8}\right)$ in example 5.1, $\mathscr{C}_{\mathscr{E}} \mathscr{E}_{1}=$ $\{-i i,-j j,-k k\}=\mathscr{C} \mathscr{E}_{-1}$.

Remark 5.1. Every undirected edge of the group $\Gamma$ in a C-graph $G$ is a Connector Edge of the vertex $i$, where $i$ is the vertex corresponding to the identity element of the group, that is $\mathscr{C} \mathscr{E}_{i}=E$.

Definition 5.3. For the C-graph $G=\left(\Gamma, E, E^{\prime}\right)$ of the group $\Gamma$, define $\Gamma_{0}=\left\{z \in \Gamma: \mathscr{C}_{\mathscr{E}_{z}}=\emptyset\right\}$. That is, $\Gamma_{0}$ is the set of vertices with empty $C E$ Set.

Example 5.3. For $G\left(Q_{8}\right)$ in example 5.1, $\Gamma_{0}=$ $\{i,-i, j,-j, k,-k\}$.

Definition 5.4. For any two distinct vertices $x$ and $y$ of the C-graph $G$ of the group $\Gamma$, if $x y$ is an undirected edge in $G$, then define the set $\mathscr{C} \mathscr{O} \mathscr{N} \mathscr{P} \mathscr{T}_{x y}=\left\{z \in \Gamma: z=C_{o N P T}, z \neq i\right\}$ and is called CoNPT Set of $x$ and $y$.

Example 5.4. In $G\left(Q_{8}\right)$ of example 5.1, $\mathscr{C} \mathscr{O} \mathscr{N} \mathscr{P} \mathscr{T}_{-i i}=\{1,-1\}=\mathscr{C} \mathscr{O} \mathscr{N} \mathscr{P} \mathscr{T}_{-j j}=$ $\mathscr{C} \mathscr{O} \mathscr{N} \mathscr{P} \mathscr{T}_{-k k}$.
Note 5.2. [1] A cyclic group with only one generator can have at most 2 elements.

Theorem 5.1. Let $G$ be the C-graph of the group $\Gamma$ with $|\Gamma| \geq 2$ and $z \in \Gamma, z \neq i$. Suppose that $z \in \Gamma_{0}$. Then the following occurs.

1. If $\Gamma$ is cyclic, then $z$ is a generator of $\Gamma$

2. If $\Gamma$ is noncyclic, then $\langle z\rangle$ is a maximal cyclic subgroup of $\Gamma$.

Proof. 1. Let the group $\Gamma$ is cyclic. By using Note 5.2 and the assumption $|\Gamma| \geq 2$, we have $\Gamma$ has atleast two distinct generators $x$ and $y$. Now, consider the given $z \in \Gamma_{0}$, then $\mathscr{C} \mathscr{E}_{z}=\emptyset$. Suppose that, $z$ is not a generator of $\Gamma$, then $\langle z\rangle \subset\langle x\rangle=\langle y\rangle$. Thus, in the C-graph $G, z \longrightarrow x$ and $z \longrightarrow y$ are two directed edges and $x y$ is an undirected edge, which implies $x y$ is a Connector Edge of the vertex $z$. That is, $x y \in \mathscr{C} \mathscr{E}_{z}$, which is a contradiction to the fact that $\mathscr{C} \mathscr{E}_{z}=\emptyset$. Therefore, $z$ is a generator of $\Gamma$.

2. Let $\Gamma$ is noncyclic and consider the given $z \in \Gamma_{0}$, then $\mathscr{C} \mathscr{E}_{z}=\emptyset$. Suppose that $x \in \Gamma$ be such that $\langle z\rangle \subset\langle x\rangle$, then clearly $\langle z\rangle \subset\left\langle x^{-1}\right\rangle$, but $\langle x\rangle=\left\langle x^{-1}\right\rangle$ and $x \neq x^{-1}$ (since if $x=x^{-1}$, then $o(x)=2 \Longrightarrow$ $o(z)=1 \Longrightarrow z=i$, a contradiction), which implies $z \longrightarrow x$ and $z \longrightarrow x^{-1}$ are two directed edges and $x x^{-1}$ is an undirected edge in $G$, this makes $x x^{-1}$ a Connector Edge of the vertex $z$. It is a contradiction to the fact that $\mathscr{C} \mathscr{E}_{z}=\emptyset$. Thus $\langle z\rangle$ is a maximal cyclic subgroup of $\Gamma$.

Definition 5.5. For the C-graph $G$ of the group $\Gamma$, define the set $\mathscr{C} \mathscr{E}_{G}=\{w \in E: w=$ $C E_{z}$ for some $\left.z \in \Gamma, z \neq i\right\}$. That is, $\mathscr{C}_{\mathscr{E}} G$ is the collection of those undirected edges of $G$ which are Connector Edges of atleast one non identity 
vertex in $G$, and is called the Connector Edge Set or $C E$ Set of the C-graph $G$.

Example 5.5. In $G\left(Q_{8}\right)$ of example 5.1, $\mathscr{C} \mathscr{E}_{Q_{8}}=$ $\{-i i,-j j,-k k\}$.

Note 5.3. $\mathscr{C}_{\mathscr{E}} \mathscr{E}_{G}=\cup_{z \in \Gamma} \mathscr{C}_{\mathscr{E}_{z}}$

Theorem 5.2. [1] The order of an element of a finite group divides the order of the group.

Theorem 5.3. (Cauchy's theorem) [1] Let $\Gamma$ be a finite group and let $p$ divides $|\Gamma|$. Then $\Gamma$ has an element of order $p$ and, consequently, a subgroup of order $p$.

Theorem 5.4. For a finite group $\Gamma$ and $\mathrm{C}$-graph $G=\left(\Gamma, E, E^{\prime}\right), \mathscr{C}_{\mathscr{E}} G=E$ if and only if $|\Gamma|=2^{n}$, $n \in \mathbb{N}$

Proof. Suppose that $\mathscr{C} \mathscr{E}_{G}=E$

Case 1: $E=\emptyset$, then for any $x, y \in \Gamma,\langle x\rangle=\langle y\rangle$ $\Longrightarrow x=y$.

But $\forall x \in \Gamma,\langle x\rangle=\left\langle x^{-1}\right\rangle \Longrightarrow x=x^{-1}, \forall x \in \Gamma$. That is, $o(x)=2 \forall x \in \Gamma \Longrightarrow 2$ divides $|\Gamma|$, by Theorem 5.2. Thus $|\Gamma|=2 k$ for some $k \in \mathbb{N}$. Now, suppose that $p \neq 2$ is a prime number dividing $|\Gamma|$, then by Theorem 5.3 , there is an element $y$ in $\Gamma$ of order $p$, which is a contradiction, since order of every element in $\Gamma$ is 2 . Therefore, $|\Gamma|=2^{n}$ for some $n \in \mathbb{N}$.

Case $2: E \neq \emptyset$, then $\mathscr{C} \mathscr{E}_{G} \neq \emptyset$. First we prove that 2 divides $|\Gamma|$. For this what we need is to show that there is at least one element in $\Gamma$ of order 2. Now $\mathscr{C} \mathscr{E}_{G} \neq \emptyset \Longrightarrow \exists$ some $x, y \in \Gamma$ such that $x y \in \mathscr{C} \mathscr{E}_{G}$. Then by the definition of the set $\mathscr{C} \mathscr{E}_{G}, \exists$ some $z_{1} \in \Gamma$ such that $z_{1} \longrightarrow x$ and $z_{1} \longrightarrow y$ are two directed edges in $G$. For this $z_{1}$, if $z_{1}=z_{1}^{-1}$, then $o\left(z_{1}\right)=2$. If $z_{1} \neq z_{1}^{-1}$, but $\left\langle z_{1}\right\rangle=\left\langle z_{1}^{-1}\right\rangle \Longrightarrow z_{1} z_{1}^{-1} \in E=\mathscr{C}_{G}$. Therefore, $\exists$ some $z_{2} \in \Gamma$ such that $z_{2} \longrightarrow z_{1}$ and $z_{2} \longrightarrow z_{1}^{-1}$ are two directed edges. By continuing this and using the fact that the group $\Gamma$ is finite, we will get a $z_{m} \in \Gamma$, such that $z_{m} \longrightarrow z_{m-1}$ and $z_{m} \longrightarrow z_{m-1}^{-1}$ are two directed edges and $z_{m}=z_{m}^{-1}$. ie, $o\left(z_{m}\right)=2$. Thus 2 divides $|\Gamma| \Longrightarrow$ $|\Gamma|=2 k$ for some $k \in \mathbb{N}$. Suppose $p \neq 2$ is a prime dividing $|\Gamma|$, then by Theorem 5.3 , there is an element $a \in \Gamma$ such that $o(a)=p \neq 2$.

Now, $\langle a\rangle=\left\langle a^{-1}\right\rangle$ and $a \neq a^{-1}$, since $o(a) \neq 2$. ie, $a a^{-1} \in E=\mathscr{C} \mathscr{E}_{G}$. Thus $\exists z \in \Gamma, z \neq i$ such that $z \longrightarrow a$ and $z \longrightarrow a^{-1}$ are two directed edges in $G$.

$$
\begin{aligned}
& \Longrightarrow\langle z\rangle \subset\langle a\rangle \text { and }\langle z\rangle \subset\left\langle a^{-1}\right\rangle . \\
& \Longrightarrow o(z) \text { divides } o(a) \text { and } o(z) \neq o(a) . \\
& \Longrightarrow o(z)=1, \text { since } o(a)=p, \text { a prime. } \\
& \Longrightarrow z=i, \text { which is a contradiction. }
\end{aligned}
$$

Therefore,

the only prime number dividing $|\Gamma|$ is 2 .

Hence, $|\Gamma|=2^{n}, n \in \mathbb{N}$.

Conversely, Suppose that $|\Gamma|=2^{n}, n \in \mathbb{N}$.

If $E=\emptyset$, then there is nothing to prove.

Suppose that $E \neq \emptyset$. We already have, $\mathscr{C} \mathscr{E}_{G} \subset$ $E$, so it is enough to prove that $E \subset \mathscr{C}_{\mathscr{E}} G$. Let $x y \in E$ which implies $\langle x\rangle=\langle y\rangle \subseteq \Gamma$ and $x \neq y$. Therefore, $|\langle x\rangle|=|\langle y\rangle|=2^{m}, 2 \leq m \leq n$ (since, if $m=1,|\langle x\rangle|=|\langle y\rangle|=2 \Longrightarrow\langle x\rangle=\{i, x\}=$ $\{i, y\}=\langle y\rangle \Longrightarrow x=y)$.

ie, $|\langle x\rangle|=|\langle y\rangle| \geq 2^{2}=4$. Thus $\exists$ at least one $z \in\langle x\rangle=\langle y\rangle$ other than $x, y$ and $i$ with $\langle z\rangle \subset\langle x\rangle=\langle y\rangle$, which implies $z \longrightarrow x$ and $z \longrightarrow y$ are two directed edges and $x y$ is an undirected edge in $G$. That is, $x y$ is a Connector Edge of $z \neq i$ in $G$. Hence, $x y \in \mathscr{C}_{\mathscr{E}} G \Longrightarrow E \subset \mathscr{C}_{\mathscr{E}}$. Thus, $\mathscr{C}_{\mathscr{E}} G=E$.

Theorem 5.5. For a group $\Gamma$ and C-graph $G=$ $\left(\Gamma, E, E^{\prime}\right), \mathscr{C}_{\mathscr{E}} G=\emptyset$ if and only if every non identity element of $\Gamma$ is of prime order.

Proof. Suppose that $\mathscr{C} \mathscr{E}_{G}=\emptyset$, then $\mathscr{C} \mathscr{E}_{z}=\emptyset$ $\forall z \in \Gamma$.

case $1: \Gamma$ is cyclic, then by theorem $5.1 z$ is a generator of $\Gamma, \forall z \in \Gamma$. That is, every non identity element of $\Gamma$ is a generator of $\Gamma$. Thus, $\Gamma \cong \mathbb{Z}_{p}$, for some prime $p$. This implies, every non iden- 
tity element of $\Gamma$ is of prime order.

case $2: \Gamma$ is non cyclic, then by theorem $5.1\langle z\rangle$ is a maximal cyclic subgroup of $\Gamma \forall z \in \Gamma$. Suppose, $\exists z \in \Gamma, z \neq i$ such that $o(z)=n$, a composite number. Let $d$ be a prime such that, $d \mid n$, then there exist an element $y \in \Gamma$ such that $o(y)=d$ and $\langle y\rangle \subset\langle z\rangle$, which is a contradiction, since $\langle y\rangle$ is maximal. Therefore, $o(z)$ is prime. That is, every non identity element of $\Gamma$ is of prime order. Conversely, suppose that every non identity element of $\Gamma$ is of prime order. Let $x y \in \mathscr{C}_{\mathscr{E}}{ }_{G} \Longrightarrow$ $\exists z \in \Gamma$ such that $z \neq i,\langle x\rangle=\langle y\rangle,\langle z\rangle \subset\langle x\rangle$ and $\langle z\rangle \subset\langle y\rangle$. This means $o(z)$ divides $o(x)$, which is a contradiction. Hence, $\mathscr{C} \mathscr{E}_{G}=\emptyset$.

Theorem 5.6. For a cyclic group $\Gamma$ and C-graph $G$, the following are true

1. If $\Gamma$ is of prime order, then $\mathscr{C} \mathscr{E}_{G}=\emptyset$.

2. If $|\Gamma|$ is a composite number and $\Gamma$ has $k$ generators, then $\left|\mathscr{C}_{G}\right| \geq \frac{k(k-1)}{2}$.

Proof. Consider a cyclic group $\Gamma$

1. Let $|\Gamma|=p$, a prime, then $\Gamma \cong \mathbb{Z}_{p} \Longrightarrow$ $o(x)=p \forall x \in \Gamma, x \neq i$. Therefore, by Theorem $5.5, \mathscr{C} \mathscr{E}_{G}=\emptyset$.

2. Suppose that $|\Gamma|=n$, a composite number. Let $X=\left\{x_{1}, x_{2}, \ldots, x_{k}\right\}$ are $k$ generators of $\Gamma$. Let $p$ be a prime dividing $n$, then Theorem 5.3 assures the existence of an element $x \neq i$ in $\Gamma$ with $o(x)=p$. Clearly $x \in \Gamma-X$. For any $i, j=1,2, \ldots, k, i \neq j$, $x_{i} x_{j}$ is an undirected edge in $G$ and also $\langle x\rangle \subset\left\langle x_{i}\right\rangle$ and $\langle x\rangle \subset\left\langle x_{j}\right\rangle$.

$\Longrightarrow x \longrightarrow x_{i}$ and $x \longrightarrow x_{j}$ are two

directed edges in $G$.

$$
\Longrightarrow x_{i} x_{j} \in \mathscr{C} \mathscr{E}_{G} \text {. }
$$

ie, corresponding to any two distinct elements in $X$, there is a member of $\mathscr{C} \mathscr{E}_{G}$. Thus, $\left|\mathscr{C}_{G}\right| \geq k C_{2}=\frac{k(k-1)}{2}$.

\section{Discussion}

The problem proposed in this paper is to construct a new graph, corresponding to groups, which ensures more clarity in the communication channel between the vertices, by assuring uniqueness in the definition from the existing graphical representations of groups. We defined it as a mixed graph, so that the one way and two way communication between the vertices, gives clarity in the graphical representation of the group, which is the main objective of this research. Since, every element of the group should be studied further, the vertex set of the graph is fixed as the group itself. Now, the main question which we dealt in the early stage of this construction is 'What is the key role of an element in the group which distinguishes it from other elements?'. In a group theoretical view, it can be the order of each element, or more clearly, it can be the set of all elements generated by an element. This character of an element in the group is the base for adjacency relation between the vertices of the graph proposed here. Then, the structural properties of the group are analysed using graph theory with some examples. More properties can be studied in this manner, which will be the scope of future work.

\section{Conclusion}

$\mathrm{C}$-graph is a mixed graphical representation of groups, which gives emphasis on the property of an element to generate other elements. The directed edges to all the non identity elements from the identity, reflect the universality of the identity element. Order of every element in a finite group can be easily deduced from the C-graph itself. By using this, it is possible to find out elements of prime order in a finite group. The Connector Edges defined for each vertex carries certain properties of the corresponding vertex such as whether it is a generator of the group or not. The Connector Edges mainly depends on the order of the 
group and in particularly order of each element in the group. More specifically, every undirected edge is a Connector Edge of at least one non identity element, only if cardinality of the group is $2^{n}$ for some $n \in N$.

\section{Aknowledgement}

The authors would like to thank University Grants Commission, India for the financial support. We would also like to thank the reviewers for their valuable suggestions and feed back, which improved the quality of research.

\section{Appendix}

\section{Chart showing identification of comparable elements in a group $\Gamma$ from the corresponding C-graph $G$.}
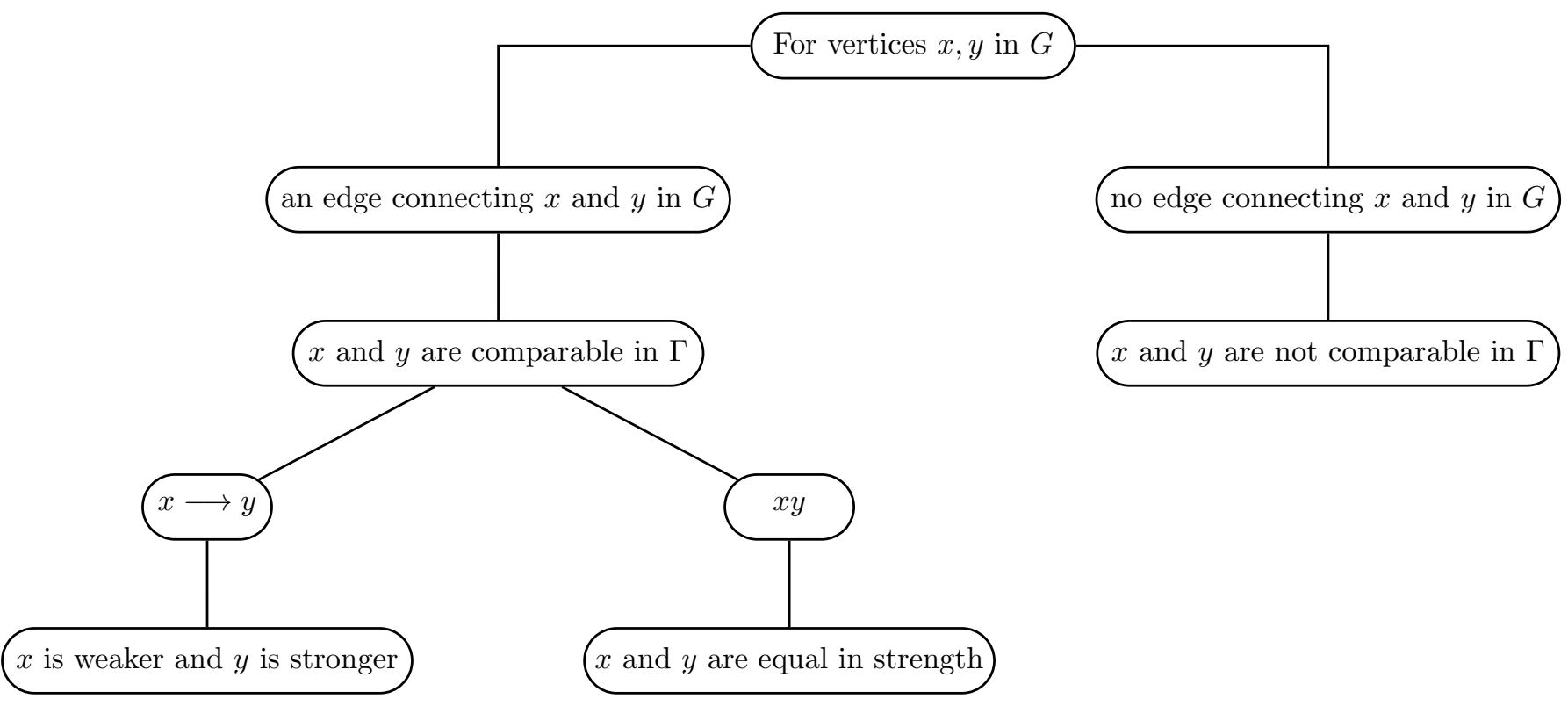

\section{References}

[1] J.B.Fraliegh, A first course in Abstract Algebra, Pearson Education, 1967.

[2] Alain Bretto, Alain Faisant, Luc Gilibert, Ggraphs A new representation of groups, journal of Symbolic Computation, 42(2007), 549560 .

[3] B.H.Neumann, A problem of Paul Erdös on Groups, Journal of the Australian Mathematical Society, 21(1976), 467-472.

[4] I.Beck, Coloring of Commutative Rings, Journal of Algebra, 116(1988), 208-226.

[5] A.Abdollahi, S.Akbari, H.R.Maimani, Non commuting Graph of a Group, Journal of Algebra, 298(2006), 468-492.
[6] A.V.Kelarev and S.J.Quinn, A combinatorial Property and Power graphs of Semigroups, Commentationes Mathematicae Universitatis Cardinae, 45(2004), 1-7.

[7] A.V.Kelarev and S.J.Quinn, Directed Graphs and Combinatorial Properties of Semigroups, Journal of Algebra, 251(2002), 16-26.

[8] Ivy Chakravarty, Shamik Ghosh and M.K.Sen, Undirected Power graphs of Semigroups, Semigroup Forum, 78(2009), 410-426.

[9] Shani P.Redmond, The zero divisor graph of a Non commutaive ring, International Jour- 
nal of Commutative Algebra, 1(2002)4, 203211.

[10] David F.Anderson, Ayman Badawi, The total graph of a commutative ring, Journal of Algebra, 320(2008)2706-2719.

[11] Kazem Khashyarmanesh, Mahdi Riza Khorsandi, Projective total graphs of commutative rings, Rocky Mountain Journal of Mathematics, 43(2013)3.

[12] Floretin Smarandache, W.B Vasantha Kandaswamy, Groups as graphs, Mathematics and Statistics Faculty and Staff Publications, 2009.

[13] M.I Sowaity, B.Sarada and A.M Naji, The identity graph of a multigroup, Far East Journal of Mathematical Sciences, $117(2019) 1,67-86$.

[14] Maria Vianney, Any Herawati, Priscila Seplinia, Henryanti, Ricky Aditya, Identity graph of a finite cyclic group, International Journal of Applied Sciences and Smart technologies, 3(2021)1.

[15] Ahmed Erfanian, Behnaz Tolue, Relative non nil-n graphs of finite groups, Science Asia, 38(2012), 201-206.

[16] P.Niroomand, A.Erfanian, M.Parvizi, B.Tolue, Non-Exterior Square Graph of Finite group, Filomat, 31(2017)3, 877-883.

[17] G.Aalipur, S.Akbari, P.J Cameron, R.Nikandish, F.Shaveisi, On the tructure of the power graph and enhanced power graph of a group, Electron.J.Combin, 24(2017), no.3, paper 3-16, 18pp.

[18] S.Bera, A.K Buniya, On enhanced power graph, J.Algebra Appl, 17(2018), no.8, 8pp.

[19] S.Zahirovic, I.Bosnjak, R.Madarasz, Enhanced Power graph of Finite groups, 2018, arXiv:1810.07627v1.

[20] S.Banerjee, On a new graph defined on the order of elements of a finite group, Preprint(2019), Available at https:|| arxiv.org | abs | 1911.02763.

[21] Xuanlong Ma, Zhonghua Way, The Coprime order graph associated with a finite group, arxiv:2011,13547v1.

[22] A.M.Mohammed, Mixed graph representation and mixed graph isomorphism, Gazi University Journal of Science,30(2017), 303310.

[23] Frank Harary, Graph Theory, Narosa Publishing House(1988).

[24] P.J.Cameron, The Power graph of a finite group ii, Journal of Group Theory, 13(2010).

\section{Creative Commons Attribution}

\section{Licence 4.0 (Attribution 4.0}

\section{International, CC BY 4.0)}

This article is published under the terms of the Creative Commons Attribution License 4.0 https://creativecommons.org/licences/by/4.0/deed.en_ US 